\title{
JAK/STAT3 and Smad3 activities are required for the wound healing properties of Periplaneta americana extracts
}

\author{
QIN SONG ${ }^{1}$, YUXIN XIE ${ }^{2}$, QIHENG GOU ${ }^{3}$, XIAOQIANG GUO $^{1}$, QIAN YAO $^{1}$ and XIAOJUN GOU ${ }^{1}$ \\ ${ }^{1}$ College of Pharmacy and Bioengineering, Chengdu University, Chengdu, Sichuan 610106; ${ }^{2}$ Cancer Center, West China Hospital, \\ Sichuan University; ${ }^{3}$ State Key Laboratory of Biotherapy/Collaborative Innovation Center of Biotherapy, \\ West China Hospital, Sichuan University, Chengdu, Sichuan 610041, P.R. China
}

Received July 6, 2016; Accepted June 13, 2017

DOI: $10.3892 /$ ijmm.2017.3040

\begin{abstract}
Periplaneta americana extracts (PAEs) play a crucial role in skin wound healing. However, their molecular effects and signaling pathways in regenerating tissues and cells are not clear. In this study, we refined the PAE from Periplaneta americana to investigate the mechanisms underlying skin wound healing. The human keratinocyte line HaCaT was selected and a mouse model of deep second-degree thermal burn was established for in vitro and in vivo studies, respectively. PAE treatment induced the proliferation and migration of $\mathrm{HaCaT}$ cells and wound healing in the burn model. Furthermore, the effects of PAE on wound healing were found to depend on the Janus-activated kinase/signal transducer and activator of transcription 3 (JAK/STAT3) pathway and Smad3 activities, according to western blot analysis and immunohistochemical (IHC) assays in vitro and in vivo. Pretreatment with a STAT3 inhibitor blocked the cell proliferation and migration induced by PAE. The results indicate the wound-healing function of PAE via enhanced JAK/STAT3 signaling and Smad3 activities. Our studies provide a theoretical basis underlying the role of PAE in cutaneous wound healing.
\end{abstract}

\section{Introduction}

Periplaneta americana (Linnaeus) (Blattodea: Blattidae), the largest insect member of the family Blattidae, is one of the most ancient insect groups with the strongest vitality. We explored its bioactive substances and the unique physiological

Correspondence to: Professor Xiaojun Gou, College of Pharmacy and Bioengineering, Chengdu University, Chengdu, Sichuan 610106, P.R. China

E-mail: 402052973@qq.com

Abbreviations: PAEs, Periplaneta americana extracts; JAK/STAT3, Janus-activated kinase/signal transducer and activator of transcription 3; IHC, immunohistochemical staining; TCM, traditional Chinese medicine; ECM, extracellular cell matrix

Key words: Periplaneta americana extracts, Janus-activated kinase, signal transducer and activator of transcription 3, Smad3, wound healing, signaling pathway mechanism. P. americana has been used in traditional Chinese medicine (TCM) to eliminate stagnant blood, clear hematocele, detoxify and promote urination and detumescence (1). P.americana has also been exploited as an alternative naturopathic remedy for impaired healing of ulcers, burn wounds, tuberculosis, ulcerative colitis, heart disease and cancer (2-6). The major focus of this study is on the promotion of wound healing.

In the early $1980 \mathrm{~s}, \mathrm{~W}_{11}-\mathrm{a}_{12}$, also known as Kangfuxin, was refined from the alcohol extract of P. americana (PAE), and was effective against thermal lesions, such as burns and scalds $(1,7)$. PAE derived from $P$. americana dry worm contains peptides, polyols, epidermal growth factors, sticky sugar acids, amino acids and other active substances (8). Recent studies have explored the pharmacological effects and mechanisms of PAE in wound healing. Histopathological studies suggest that the necrotic tissue and inflammatory exudate induced by thermal lesions or chemical damages were significantly reduced after PAE treatment in rat and rabbit models (9). In addition, increased epithelial repair area, follicular regeneration, early fibrous tissue repair and rapid decrease of inflammatory cells were detected in the PAE-treated groups. PAE also promoted the synthesis and secretion of extracellular cell matrix (ECM) in the wound (10); activated cellular functions by accelerating the opening of macrophage ion channels (11); increased the number of neutrophils, improved the granulocyte chemotactic function and spontaneous movement, resulting in wound repair and clearance $(12,13)$.

However, wound healing is a complex process of cell proliferation, migration, matrix synthesis and contraction, and involves various types of cells and regulatory mechanisms. Resident cells (keratinocytes, fibroblasts and endothelial cells) and inflammatory cells participate in wound healing $(2,14)$. Evidence has revealed that several signaling pathways are associated with wound healing via triggering their target gene expression, such as the Janus-activated kinase/signal transducer and activator of transcription 3 (JAK/STAT3) signaling (15-18). In wound healing, cytokines contribute to activate STATs and the activated JAK/STAT3 pathway controls the proliferation and differentiation necessary for wound healing $(19,20)$. Furthermore, through activation of JAK/STAT3 signaling cascades, the cytokine induces anti-apoptotic pathways and anti-microbial molecules to help prevent tissue damage and aid in their repair (21-23). In addition, a study demonstrated a critical role for STAT3 in 
the migration but not proliferation of keratinocytes in wound healing (24). The pivotal roles of Smad3 signaling in cutaneous wound healing have been well documented $(15,16)$. Smad3 binds with a Smad mediator (SMAD4) to form a complex, moving into the nucleus and regulates the expression of genes including those involved in keratinocyte migration, fibroblast infiltration and extracellular matrix construction $(25,26)$. Additionally, Smad 3 could balance the reepithelialization and fibrogenesis of the repaired tissues $(27,28)$.

Previous studies have mainly focused on the effect of PAE on cellular repair (10-13). The resident biological functions and cellular signal transduction pathways underlying the healing effect of PAE are not fully established. In the present study, we investigated the biological function and mechanisms of PAE in human keratinocyte cells and rat skin injury models to facilitate the clinical application of PAE.

\section{Materials and methods}

Cell lines. The spontaneously immortalized human keratinocyte HaCaT cell line was obtained from the American Type Culture Collection (ATCC, Rockville, MD, USA) and was cultured in Dulbecco's modified Eagle's medium (DMEM) supplemented with $10 \%$ fetal calf serum (FCS) and $1 \%$ penicillin-streptomycin. Culture conditions were maintained constant at $37^{\circ} \mathrm{C}$ in a $5 \% \mathrm{CO}_{2}$ humidified atmosphere.

Preparation of PAE. P. americana was obtained from the Good Agricultural Practice (GAP) breeding base (Sichuan, China). P. americana (200 g) powder was extracted with $90 \% \mathrm{EtOH}\left(1.2\right.$ liters) twice at $80^{\circ} \mathrm{C}$. After solvent evaporation, the ethanol extract was recovered. The extract $(20 \mathrm{~g})$ was suspended in water $(200 \mathrm{ml})$ at $80^{\circ} \mathrm{C}$ and filtered through a $0.22-\mathrm{mm}$ filter membrane in appropriate concentrations and stored at $-20^{\circ} \mathrm{C}$ until use. HPLC-diode array detector (HPLC-DAD) was used for the study of P.americana extract. Diamonsil C18 (250x4.6 mm; $5 \mu \mathrm{m})$ was selected as the chromatography column. The optimized mobile phase consisted of solvent A (3\% v/v methanol in water containing $0.07 \% \mathrm{v} / \mathrm{v}$ acetic acid) and solvent $\mathrm{B}$ (methanol). The following gradient was used to represent time (min)/mobile phase A (\%)/mobile phase B (\%): 0.0/100/0, 10/100/0, 20/70/30, 21/50/50,35/0/100, at a flow rate of $0.6 \mathrm{ml} / \mathrm{min}$ at $25^{\circ} \mathrm{C}$, and $254 \mathrm{~nm}$ in $10 \mu \mathrm{l}$ injection volume.

In vitro proliferation, migration and wound healing assays. Proliferation was determined using the 3-(4,5-dimethylthiazol-2-yl)-2,5-diphenyltetrazolium bromide (MTT) assay. Cells were seeded in a volume of $200 \mu 1(2,000$ cells/well $)$ on 96-well plates after cultivation with or without PAE. The culture medium containing serum was replaced by MTT every $24 \mathrm{~h}$. A final MTT concentration of $0.5 \mathrm{mg} / \mathrm{ml}$ was added to the wells followed by incubation for $4 \mathrm{~h}$ at $37^{\circ} \mathrm{C}$. The supernatant was discarded and replaced with dimethyl sulfoxide (DMSO) (150 $\mu \mathrm{l} /$ well). The optical densities (OD) were measured at $570 \mathrm{~nm}$ with a microplate reader (Bio-Rad, Hercules, CA, USA). The experiment was repeated in triplicate.

Cell migration was analyzed in a 24 -well plate with Millicell Cell Culture Insert, using an $8-\mu \mathrm{m}$ pore size polycarbonate membrane (Millipore Corporation, Billerica, MA,
USA). After $48 \mathrm{~h}$ of cultivation in a medium with or without PAE $(0.3125 \mathrm{mg} / \mathrm{ml})$, the cells $\left(2 \times 10^{4} /\right.$ well $)$ were transferred into the upper chamber with the non-coated membrane and suspended in $200 \mu \mathrm{l}$ of serum-free DMEM. In the lower chamber, $600 \mu \mathrm{l}$ of the medium supplemented with $10 \%$ fetal bovine serum was added. After incubation for $24 \mathrm{~h}$, the chambers were fixed with $4 \%$ paraformaldehyde for $20 \mathrm{~min}$, and stained with hematoxylin for $15 \mathrm{~min}$. Images were captured with an optical microscope.

The wound healing assay was performed by seeding the cell cultures on a 6 -well plate and grown to $90 \%$ confluency, followed by $48 \mathrm{~h}$ of starvation in serum-free medium with or without PAE $(0.3125 \mathrm{mg} / \mathrm{ml})$. The culture medium was removed and the monolayers were scratched using a 200- $\mu$ l pipette to create a uniform cell-free wound area. Debris was removed by gently washing with sterile phosphate-buffered saline (PBS). Cell migration into the wound area was monitored and photographed at 0,24 and $48 \mathrm{~h}$ using an optical microscope.

Western blot analysis and antibodies. Cells were lysed with radioimmunoprecipitation assay (RIPA) buffer and phosphatase inhibitors. The protein concentration was measured using the bicinchoninic acid (BCA)-protein quantification assay (Beyotime Biotechnology, Shanghai, China). Normalized lysates $(30 \mu \mathrm{g})$ were separated by electrophoresis on $8-12 \%$ sodium dodecyl sulfate-polyacrylamide gel electrophoresis (SDS-PAGE) gel and transferred to a PVDF membrane (Millipore Corporation). The membrane was blocked for $1 \mathrm{~h}$ at room temperature and incubated overnight at $4^{\circ} \mathrm{C}$ with primary antibody. After three washes with TBST, the membrane was incubated with horseradish peroxidase-(HRP)-conjugated IgG. Signals were visualized with enhanced chemiluminescence (ECL; Millipore Corporation). Primary antibodies against Smad3 (\#9523), phospho-Stat3 (\#9145), Stat3 (\#12640), JAK1 (\#3344), JAK2 (\#3230) (dilution, 1:1,000; Cell Signaling Technology, Beverly, MA, USA), phospho-Smad3 (ab52903), phospho-JAK2 (ab32101) (dilution, 1:1,000; Abcam, Cambridge, MA, USA), phospho-JAK1 (sc-101716; dilution, 1:1,000; Santa Cruz Biotechnology, Inc., Dallas, TX, USA) were used.

Immunofluorescence staining. Each group of HaCaT cells was washed with PBS followed by fixation with $4 \%$ paraformaldehyde ( $\mathrm{pH} 7.4)$ in 6-well plates. Cells were incubated with $0.5 \%$ Triton $\mathrm{X}-100$ for $30 \mathrm{~min}$ at room temperature, and treated with 5\% BSA for $1 \mathrm{~h}$. Cells were incubated with the following primary antibodies: Smad3 (\#9523; dilution, 1:100), phospho-Stat3 (\#9145; dilution, 1:100), Stat3 (\#12640; dilution, 1:500) (all from Cell Signaling Technology), and phospho-Smad3 (ab52903; dilution, 1:100; Abcam) overnight at $4^{\circ} \mathrm{C}$. The cells were incubated with the corresponding fluorescent dye-conjugated secondary antibodies (\#4412; dilution, 1:200; Cell Signaling Technology) at $37^{\circ} \mathrm{C}$ for $1 \mathrm{~h}$, and protected from light. The cells were visualized via fluorescence microscopy.

Rat skin injury models. Healthy adult C57 male mice were purchased from the West China School of Preclinical and Forensic Medicine, Sichuan University, Sichuan, China. All the experiments were conducted according to the Guide for the Care and Use of Laboratory Animals at the Animal 
Experimental Center of Sichuan University. We obtained ethical approval of the Medical Ethics Committee of Sichuan University with approval no. K2016033. Thermal injuries were created with a solid aluminum bar measuring $10 \mathrm{~mm}$ in diameter, and pre-heated in boiling water at a temperature of $100^{\circ} \mathrm{C}$. The bar was maintained symmetrically in contact with the skin on the dorsal flank for $15 \mathrm{sec}(29)$. The right skin of the dorsal flank was wiped with PAE (original fluid, $5 \mathrm{mg} / \mathrm{ml}$ ), while the left was treated with normal saline as the untreated control. The daily treatment regimen lasted for 21 days. After injury, to minimize the suffering of the animals, each mouse was housed individually and fed with sterilized food and tap water to prevent infection. The wound healing rates were measured on days $0,7,14$ and 21 after injury, and the complete wound healing time was calculated as follows: Healing rate $=($ original wound area - non-healing wound area $) /$ original wound area (30). Mice were sacrificed after 3 weeks.

Immunohistochemistry. All the immunohistochemical assays were conducted following the manufacturer's instructions. Briefly, the skin tissues were fixed in formalin and embedded in paraffin. Consecutive paraffin sections (4- $\mu \mathrm{m})$ of the tissue samples were prepared and incubated overnight at $4^{\circ} \mathrm{C}$ with primary antibodies followed by incubation with peroxidase-labeled polymer conjugated to goat anti-rabbit immunoglobulins (EnVision/HRP; Dako, Glostrup, Denmark). Primary antibodies against Smad3 (\#9523), phospho-Stat3 (\#9145), Stat3 (\#12640), JAK1 (\#3344), JAK2 (\#3230) (dilution, 1:1,000; all from Cell Signaling Technology), phospho-Smad3 (ab52903), phospho-JAK2 (ab32101) (dilution, 1:1,000; both from Abcam), phospho-JAK1 (sc-101716; dilution 1:1,000; Santa Cruz Biotechnology, Inc.) were used.

Statistical analysis. Statistical analyses were performed using SPSS 19.0 (SPSS, Inc., Chicago, IL, USA) or Prism 5.0 (GraphPad Software, La Jolla, CA, USA). Quantitative data were analyzed using a two-tailed Student's t-test, and one-way analysis of variance (ANOVA) followed by Dunnett's multiple comparison post-test. Differences were considered statistically significant at $\mathrm{P}<0.05, \mathrm{P}<0.05$ and $\mathrm{P}<0.01$.

\section{Results}

Chromatographic separation of PAE. PAE contained polyalcohols, amino acids, pyrimidines and proteoglycans. Analysis of PAE revealed the presence of uracil (retention time $7.626 \mathrm{~min}, 1.145 \mathrm{mg} / \mathrm{g}$ ), hypoxanthine (retention time $11.707 \mathrm{~min}, 4.253 \mathrm{mg} / \mathrm{g}$ ), and inosine (retention time $20.427 \mathrm{~min}, 8.156 \mathrm{mg} / \mathrm{g}$ ) (Fig. 1).

PAE increases cell proliferation and migration of human keratinocytes in vitro. The MTT assay was used to confirm the proliferation of $\mathrm{HaCaT}$ cells in the presence of PAE at three time-points. The low $(0.3125 \mathrm{mg} / \mathrm{ml})$ dose of the extract induced optimal growth in HaCaT cells at 24, 48 and $72 \mathrm{~h}$, respectively (p<0.05) (Fig. 2A). Conversely, high doses of PAE $(1.25$ and $2.5 \mathrm{mg} / \mathrm{ml})$ inhibited cell proliferation. Furthermore, the treatment with the extract caused obvious proliferation after $48 \mathrm{~h}$ of treatment. Transwell and wound healing assays were used to determine $\mathrm{HaCaT}$ cell migration in skin. Fig. 2B
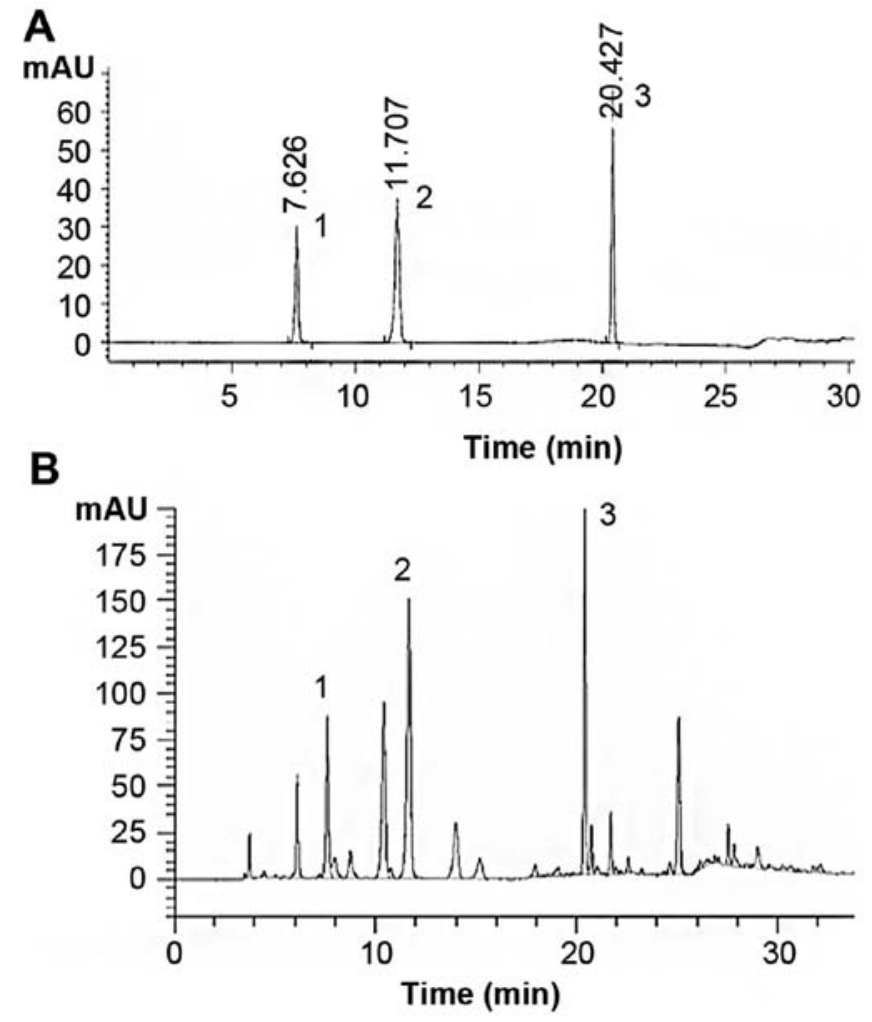

Figure 1. Chromatographic separation of Periplaneta americana extract (PAE). (A) HPLC chromatogram of authentic standards tested. (B) HPLC chromatogram of PAE at $254 \mathrm{~nm}$ : peak 1: uracil $(1.145 \mathrm{mg} / \mathrm{g})$, peak 2: hypoxanthine $(4.253 \mathrm{mg} / \mathrm{g})$, and peak 3 : inosine $(8.156 \mathrm{mg} / \mathrm{g})$. Identification was based on retention time, and UV spectra compared with commercial standards.

shows that compared with the control group, cell migration in the PAE-treated group increased significantly $(\mathrm{p}<0.01)$. Furthermore, consistent with the results from the Transwell assay, data from the wound healing assay also showed significantly improved migration of wound closure in the PAE treatment group compared with the control group in the HaCaT cells $(\mathrm{p}<0.01)$ (Fig. 2C).

PAE enhances the JAK/STAT3 signaling pathway and Smad 3 activities in human keratinocytes. To better understand the signaling pathway or mechanism underlying the PAE-mediated regulation of $\mathrm{HaCaT}$ cells, the signaling molecules associated with proliferation and migration were screened. After PAE treatment, the expression levels of p-JAK1, p-JAK2 and its downstream molecule p-STAT3 were markedly upregulated in the HaCaT cells, while the total protein levels (JAK1, JAK2 and STAT3) were unchanged $(\mathrm{p}<0.01)(\mathrm{Fig} .3 \mathrm{~A})$. In addition, phosphorylation of Smad3 (p-Smad3) was also significantly increased, while Smad3 was not affected $(\mathrm{p}<0.01)$ (Fig. 3B). However, nuclear factor- $\kappa \mathrm{B}(\mathrm{NF}-\kappa \mathrm{B}) / \mathrm{p} 65$ and $\beta$-catenin expression was not distinctly different between the PAE treatment and the control groups of HaCaT cells ( $\mathrm{p}>0.05$ ) (Fig. 3C).

Effects of STAT3 inhibitor on PAE-mediated proliferation and migration of HaCaT cells. To further confirm that PAE increases cell proliferation and migration of human keratinocytes via JAK/STAT3 signaling, the inhibitor of STAT3 

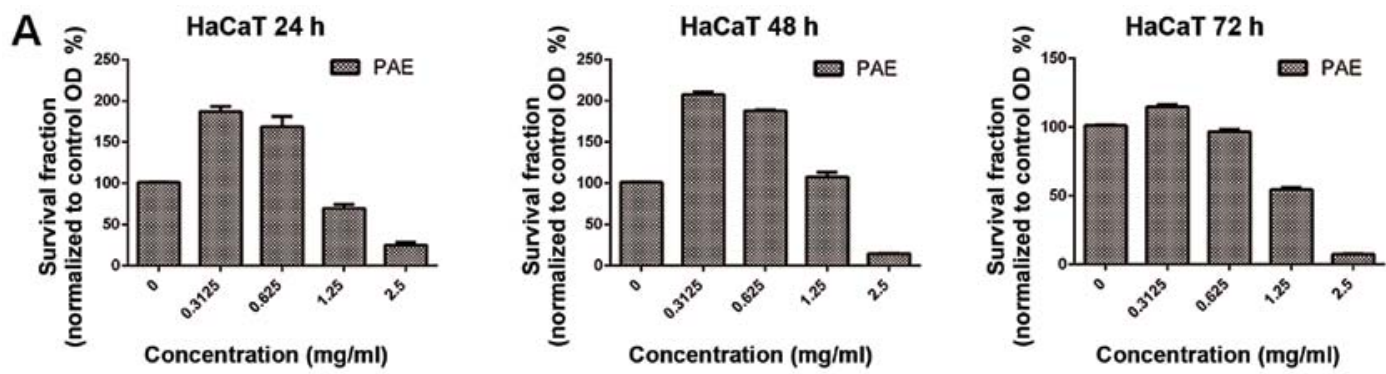

B
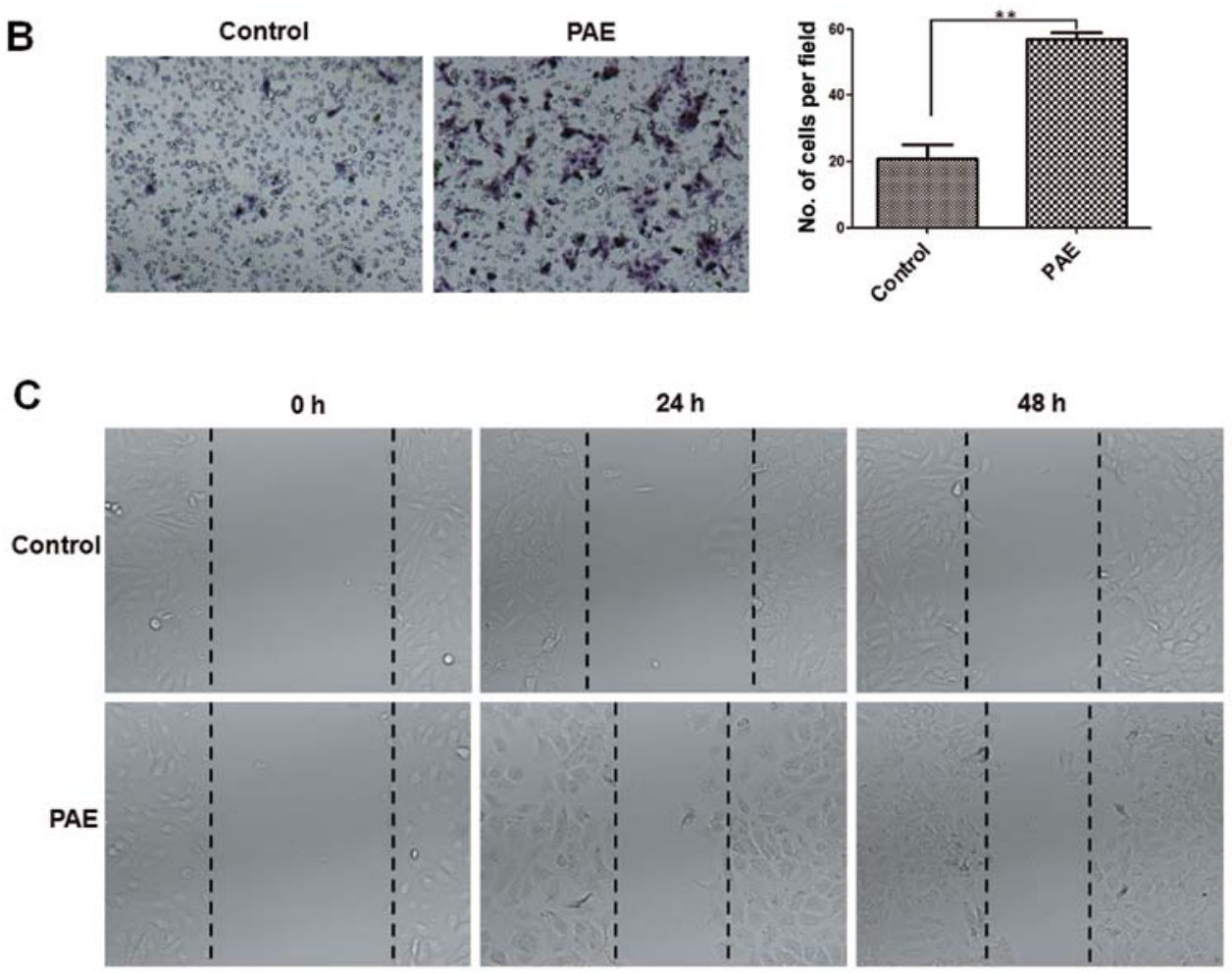

Figure 2. Effects of different doses of Periplaneta americana extract (PAE) on differentiation at different time-points and effects on migration in HaCaT cells. (A) Left: the survival fraction of HaCaT cells following treatment with different doses of the extract for $24 \mathrm{~h}$ (normalized to control group); middle: the survival fraction of $\mathrm{HaCaT}$ cells following treatment with different doses of the extract for $48 \mathrm{~h}$ (normalized to control group); right: the survival fraction of $\mathrm{HaCaT}$ cells following treatment with different doses of the extract for $72 \mathrm{~h}$ (normalized to control group). (B) HaCaT cells were treated with or without PAE $(0.3125 \mathrm{mg} / \mathrm{ml})$ for $48 \mathrm{~h}$, and cells treated without PAE served as controls. HaCaT cells were plated on Matrigel-coated membranes for migration assays and incubated for $24 \mathrm{~h}$. Migratory cells were counted in 10 random fields and expressed as the average number of cells/field. (C) Wound healing assay: images obtained at 0, 24 and $48 \mathrm{~h}$ after scratching. Data represent mean $\pm \mathrm{SD} .{ }^{* *} \mathrm{p}<0.01 \mathrm{vs}$. the control group.

was used to verify the effects of PAE on cell proliferation and migration. A STAT3 inhibitor, stattic, was used to block STAT3 activation. Phosphorylation of STAT3 was decreased after treatment with stattic in a dose-dependent manner in the HaCaT cells. However, the total expression of STAT3 was not changed. Furthermore, when cells were treated with PAE, after inhibition of STAT3 phosphorylation by stattic, the p-STAT3 levels remained unchanged (Fig. 4A and B). Furthermore, as shown in Fig. 4C and D, PAE-induced cell growth and migration were abrogated by pretreatment with static in the HaCaT cells. The result confirmed that the PAE extract promoted $\mathrm{HaCaT}$ cell proliferation and migration by activating the JAK/STAT3 signaling pathway.

PAE increases phosphorylation of STAT3 and Smad3 expression and alters their distribution. The distribution pattern of STAT3 and Smad3 was investigated using fluorescence immunostaining. The results demonstrated that PAE treatment increased STAT3 phosphorylation in the cytoplasm followed by nuclear translocation (Fig. 5A). By contrast, the total expression of STAT3 was constant irrespective of PAE stimulation in the HaCaT cells (Fig. 5B). Furthermore, phosphorylation of Smad3 (p-Smad3) was also significantly increased in PAE-treated cells, while total Smad3 remained unchanged (Fig. 5C and D). The altered phosphorylation of STAT3 and Smad3 suggested activation of the JAK/STAT3 and $\mathrm{Smad} 3$ signaling cascade by PAE in HaCaT cells.

PAE promotes wound healing in vivo. To establish the in vivo effect of PAE on cutaneous wound healing, we established a mouse model of deep second-degree thermal burn (16). Skin lesions were not prominent initially, but eventually appeared and worsened. The results demonstrated that the burn wound in the PAE (original fluid, $50 \mathrm{mg} / \mathrm{ml}$ )-treated side (right 

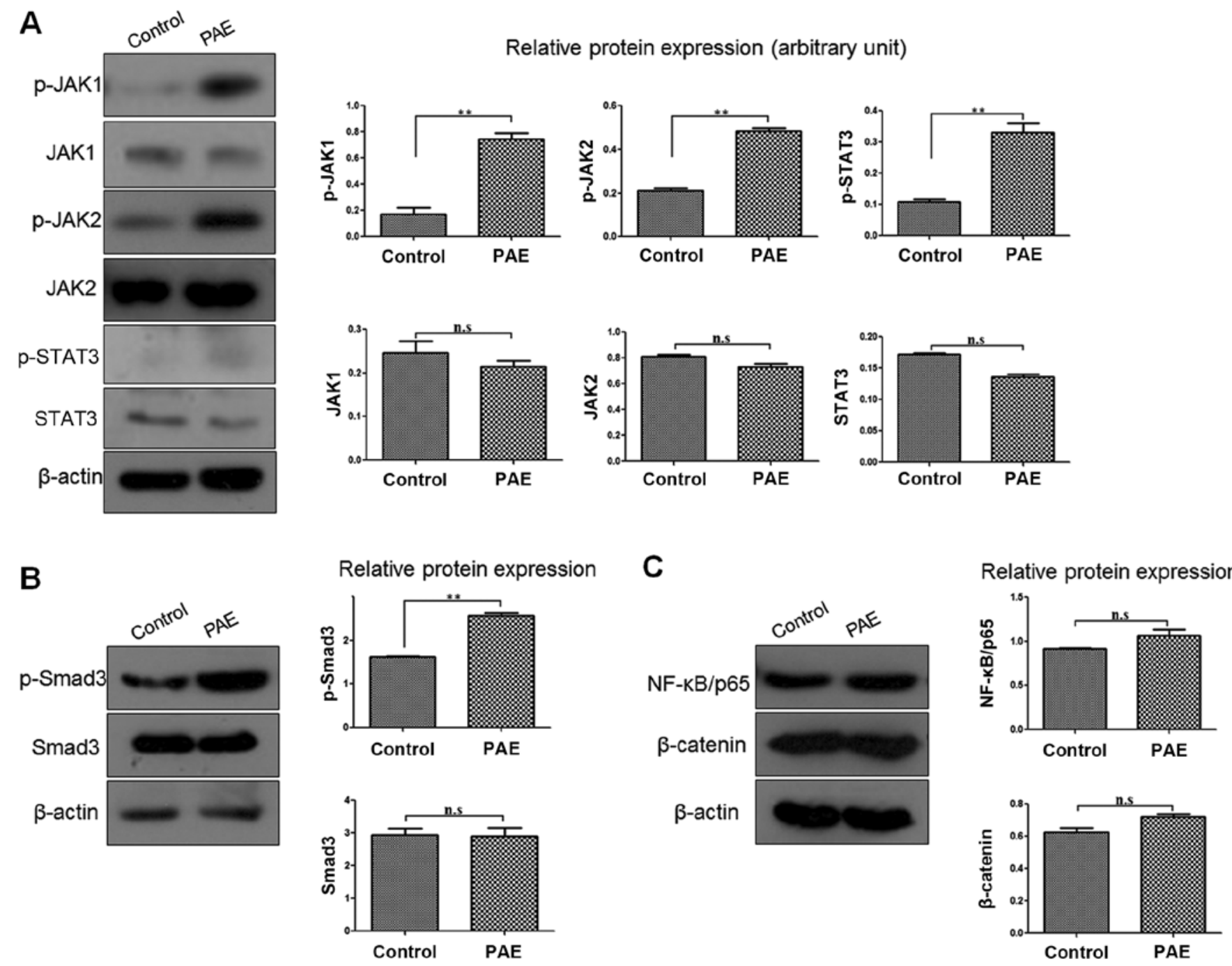

Figure 3. Effects of Periplaneta americana extract (PAE) on cell proliferation and migration-related signaling molecules in $\mathrm{HaCaT}$ cells. HaCaT cells were treated with or without PAE $(0.3125 \mathrm{mg} / \mathrm{ml})$ for $48 \mathrm{~h}$, and cells treated without PAE served as the controls. (A) The protein expression of Janus-activated kinase/signal transducer and activator of transcription 3 (JAK/STAT3) signaling pathway, (B) Smad3 and its phosphorylation, (C) NF- $\mathrm{kB} / \mathrm{p} 65$ and $\beta$-catenin were determined by western blotting. $\beta$-actin was used as a loading control. Data represent mean $\pm \mathrm{SD}$. ${ }^{* *} \mathrm{p}<0.01$ vs. the control group. n.s. not significant.

side) showed significant fibroblast proliferation and fibrosis resulting in scar tissue formation compared with the control (left side) (Fig. 6A). Histopathological analysis revealed an increase in epithelial repair, follicle regeneration and additional fibrous tissues in the PAE-treated groups (Fig. 6B). The healing rates and times of skin wounds without and with PAE treatment were sequentially recorded and evaluated. The wound healing rates were measured on days $0,7,14$ and 21 after burn, and the complete wound healing time was calculated. The healing rate of the treated group was significantly higher than that of the control group at different time-points $(\mathrm{p}<0.05)$ (Table I and Fig. 6C). The results suggest that PAE accelerates the healing of burn wounds.

JAK/STAT3 signaling and Smad3 activities in PAE-mediated wound healing. To further confirm the signaling cascade and mechanism of PAE-induced regulation of wound healing, immunohistochemical (IHC) and western blot analysis were used to assay the JAK/STAT3 signaling and phosphorylation of Smad3 in burn wound specimens. Enhanced nuclear staining of p-JAK1, p-JAK2, p-STAT3 and p-Smad3 was detected in the PAE treatment group compared with the control group. By contrast, the nuclear expression of JAK1, JAK2, STAT3 and Smad3 proteins showed no significant difference between the control wounds and the treated wounds (Fig. 7A). Consistent with the IHC findings, the western blot analysis further validated the increased expression of p-JAK1, p-JAK2, p-STAT3, p-Smad3, but not JAK1, JAK2, STAT3 and Smad3 proteins (Fig. 7B).

\section{Discussion}

The beneficial effects of Periplaneta americana extracts (PAEs) on skin wound healing have been adequately demonstrated, suggesting their potential role as bioactive agents for alternative treatment of skin wounds $(1,2)$. However, the role of PAE in the regulation of target cells and the molecular mechanisms of wound healing in the skin are still unclear. In the present study, we extracted and purified PAE from P. americana to explore the underlying mechanism. We established skin wound healing models in vitro and in vivo to demonstrate that 

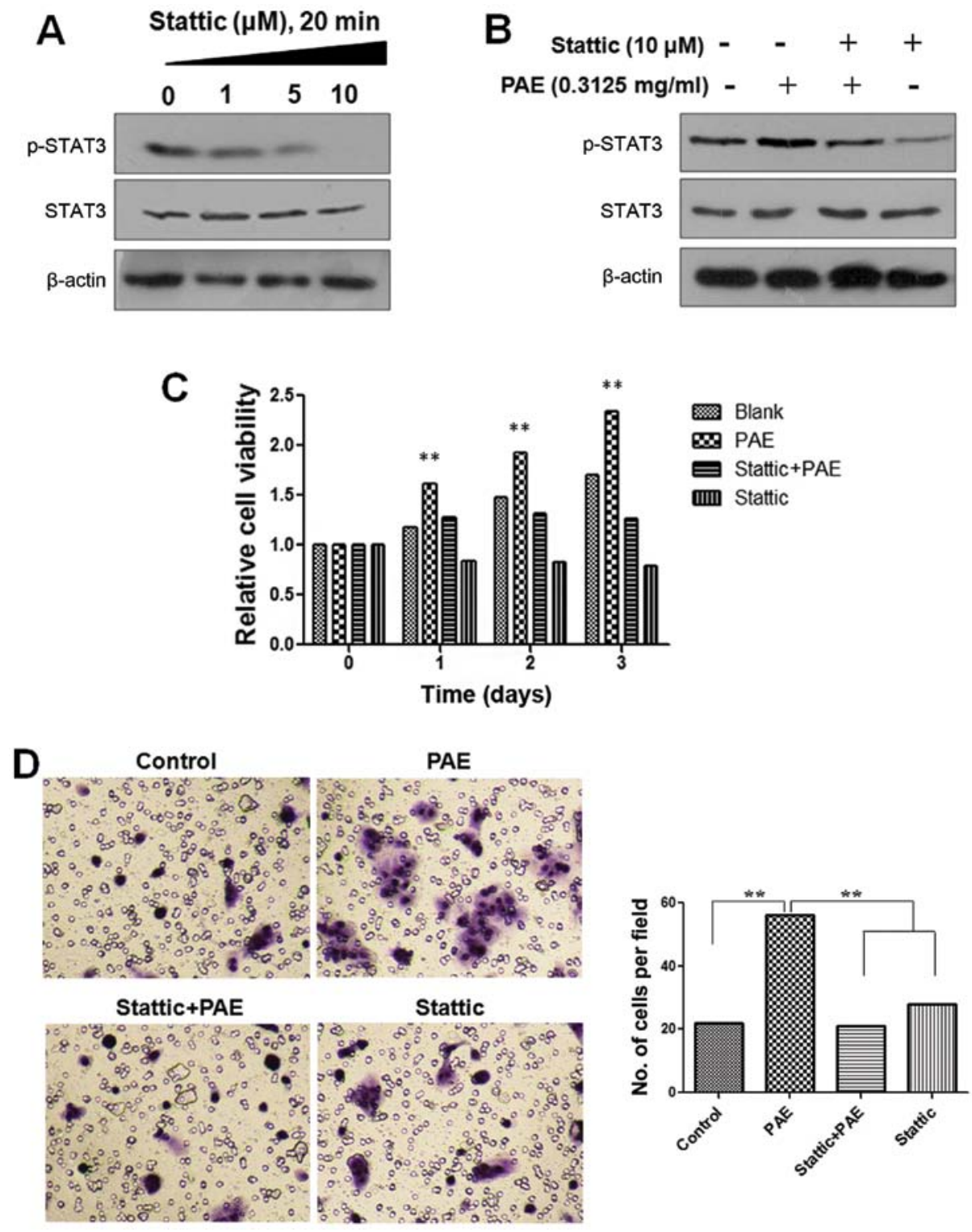

Figure 4. Effects of STAT3 inhibitor on Periplaneta americana extract (PAE)-mediated signal transduction, proliferation and migration in HaCaT cells. (A) $\mathrm{HaCaT}$ cells were pretreated with different concentrations of the STAT3 inhibitor stattic $(0,1,5$ and $10 \mu \mathrm{M})$ for 20 min, and p-STAT3, total STAT3 and $\beta$-actin were detected by western blotting. (B) HaCaT cells were incubated in the medium containing PAE at the indicated concentrations for $48 \mathrm{~h}$ after pretreatment with $10 \mu \mathrm{M}$ stattic for $20 \mathrm{~min}$. The expression of p-STAT3, total STAT3 and $\beta$-actin was detected by western blotting. HaCaT cells were incubated with a medium containing PAE at the indicated concentrations for $48 \mathrm{~h}$ after pretreatment with stattic $(10 \mu \mathrm{M})$ for 20 min. (C) Cell proliferation was determined by MTT and (D) migration was tested by Transwell assay. ${ }^{* *} \mathrm{p}<0.01$.

PAE promoted the proliferation and migration of human keratinocyte $\mathrm{HaCaT}$ cells and wound healing in a mouse model. The effects of PAE were mediated via the JAK/STAT3 and Smad3 signaling pathways in vitro and in vivo.

Wound healing comprises four distinct but overlapping phases: hemostasis, inflammation, proliferation and remodeling $(2,31)$. Keratinocytes are a major skin component and an important determinant of wound healing efficiency $(15,32,33)$. In this study, we found that PAE promoted the proliferation and migration of human immortalized keratinocyte $\mathrm{HaCaT}$ cells. To confirm the pharmacological effects of PAE and establish the optimal extraction process, the human immortalized keratinocyte line $\mathrm{HaCaT}$ was used as an in vitro wound healing model for the evaluation of the cellular and molecular effects of PAE. The results showed an increase in proliferation and migration of keratinocytes after treatment with PAE $(0.3125 \mathrm{mg} / \mathrm{ml})$ for $48 \mathrm{~h}$. In addition, PAE significantly increased the healing rates and time by enhancing the epithelial repair, follicle regeneration and fibrous tissue proliferation in cutaneous thermal burns in vivo.

Although the effects of PAE on wound healing have long been recognized, the underlying molecular mechanisms remain largely unknown. Studies suggest the role of numerous signaling pathways in cutaneous wound healing (15-18). In this study, the expression of JAK/STAT3 signaling and the activation of Smad3, NF- $\mathrm{KB} / \mathrm{p} 65$ and $\beta$-catenin in vitro and in wound tissues in vivo were evaluated by western blot analysis and validated immunohistochemically. We found that the expression and nuclear translocation of p-JAK1, p-JAK2 and its downstream p-STAT3 were markedly upregulated in the HaCaT cells and wound tissues after PAE treatment. In addition, the levels of 

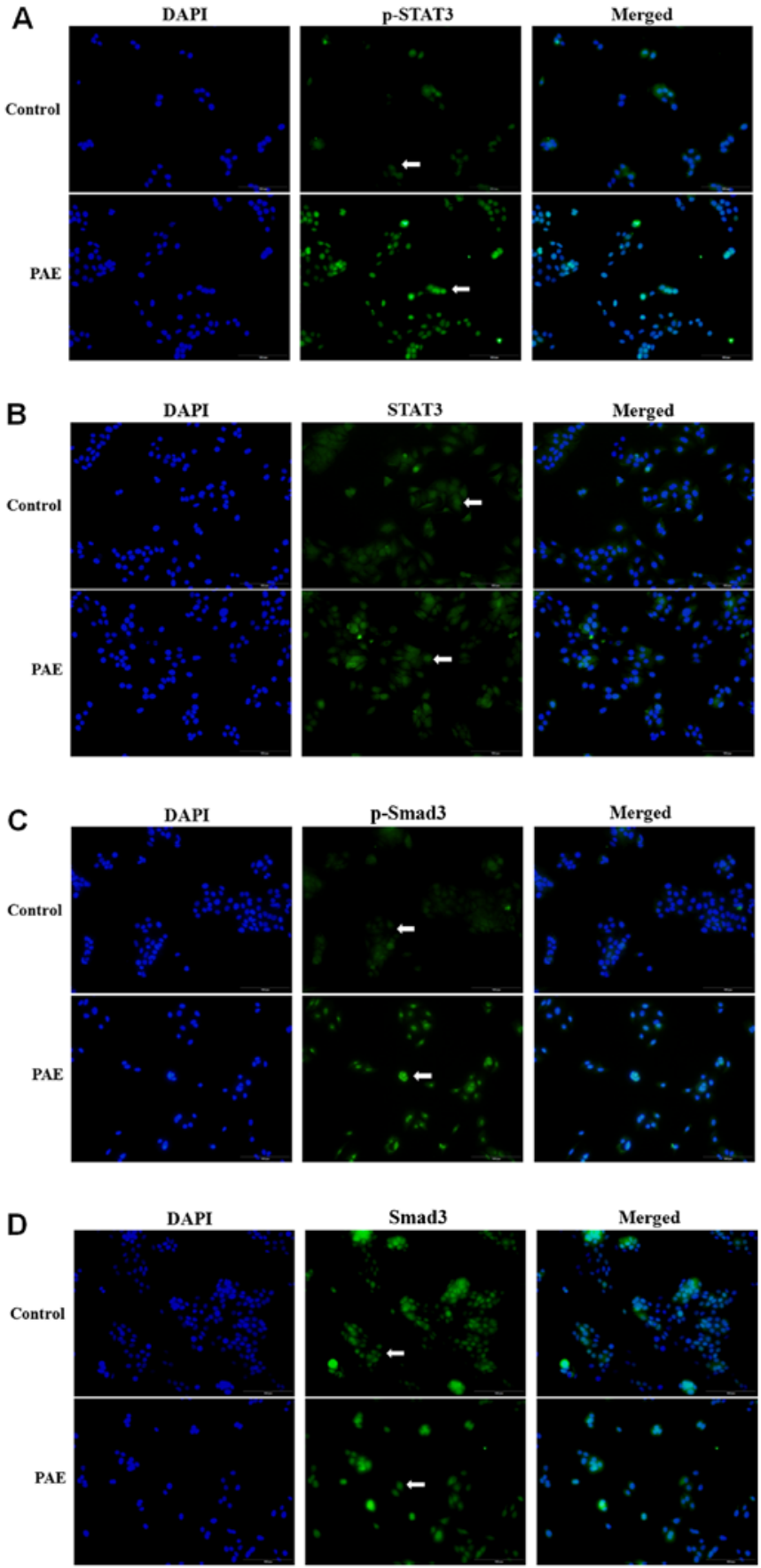

Figure 5. Immunofluorescence analysis of p-STAT3/STAT3 and p-Smad3/ Smad3 protein. Cells were treated with Periplaneta americana extract (PAE) for $48 \mathrm{~h}$, followed by fixation and incubation to determine immunofluorescence. The antibody against (A and B) p-STAT3/STAT3 and (C and D) $\mathrm{p}$-Smad3/Smad3 presented green fluorescence, whereas a blue fluorescent signal was produced by DAPI staining of the cell nuclei. Areas of overlap between the green and the blue fluorescence were observed in the merged images. The arrows in (A) indicate p-STAT3 expression in the nucleus. The arrows in (B) indicate STAT3 expression in the nucleus and cytoplasm. The arrows in (C) indicate $\mathrm{p}-\mathrm{Smad} 3$ expression in the nucleus. The arrows in (D) indicate Smad3 expression in the nucleus and cytoplasm.

p-Smad3 expression were increased in the treated cells and tissues, suggesting the active role of these signaling pathways in PAE-mediated wound healing. However, NF- $\kappa \mathrm{B}$ and Wnt signaling appeared to be minimally activated irrespective of PAE treatment because of limited expression of $\mathrm{NF}-\kappa \mathrm{B} / \mathrm{P} 65$ and $\beta$-catenin upregulation, or nuclear translocation.

Table I. Healing rates at different time-points after burn (\%).

\begin{tabular}{lccc}
\hline Group & 7 day & 14 day & 21 day \\
\hline Control & $28.17 \pm 7.03$ & $58.73 \pm 4.43$ & $87.72 \pm 2.88$ \\
Treatment & $37.34 \pm 8.39^{\mathrm{a}}$ & $83.61 \pm 4.61^{\mathrm{a}}$ & $97.69 \pm 0.82^{\mathrm{a}}$
\end{tabular}

${ }^{a}$ The healing rate of the treatment group was compared with the control group at varied time-points, $\mathrm{p}<0.05$.

A
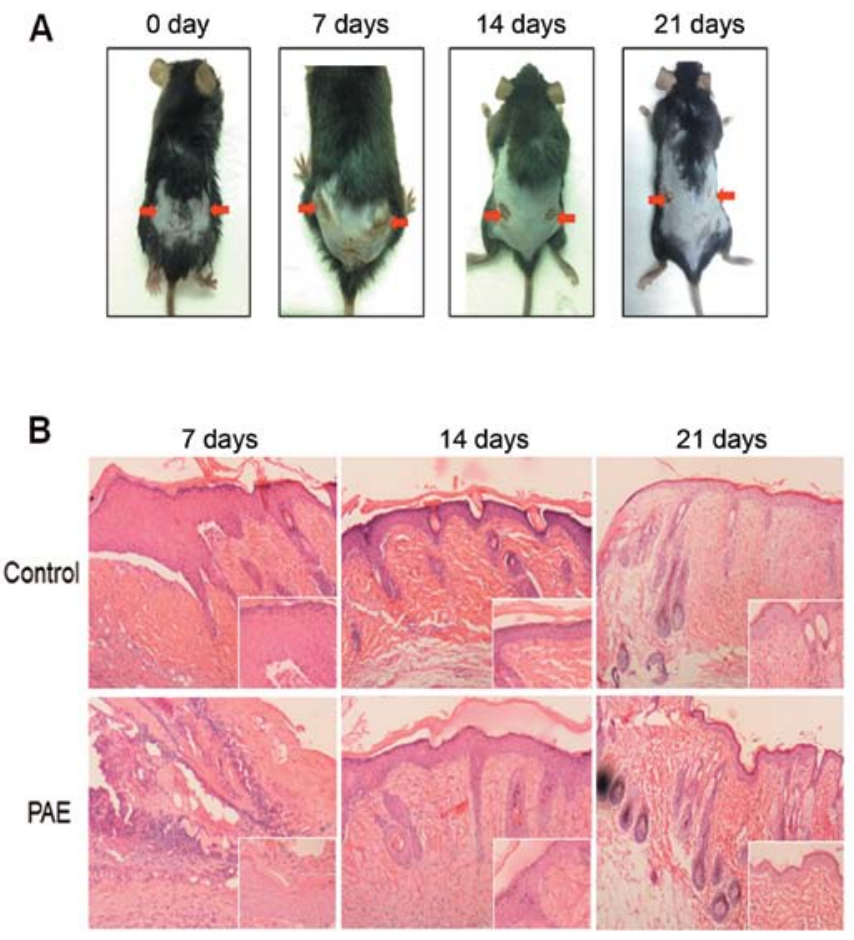

C

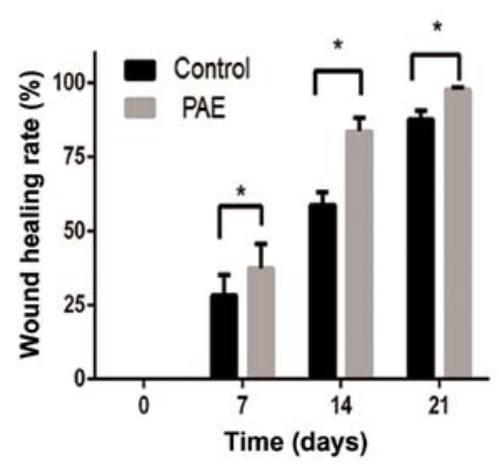

Figure 6. Effects of Periplaneta americana extract (PAE) on wound healing of skin thermal burn in vivo. In the mouse model, the right burn wound of the dorsal flank was wiped with PAE (original fluid), while the left was treated daily with normal saline. (A) Representative images of burn wounds were monitored on days $0,7,14$ and 21. (B) Histological H\&E staining of burnt tissues on days 0,14 and 21 after burn injury, respectively. (magnification, $\mathrm{x} 100$ and $\mathrm{x} 400)$. (C) The wound healing rates. " $\mathrm{p}<0.05$ vs. the control group.

STAT3 is a member of the latent transcription factor family that acts as a downstream effector of cytokine and growth factor receptor signaling. The canonical JAK/STAT signaling pathway involves the activation of JAK or growth factor receptor kinases, phosphorylation of STAT proteins, 


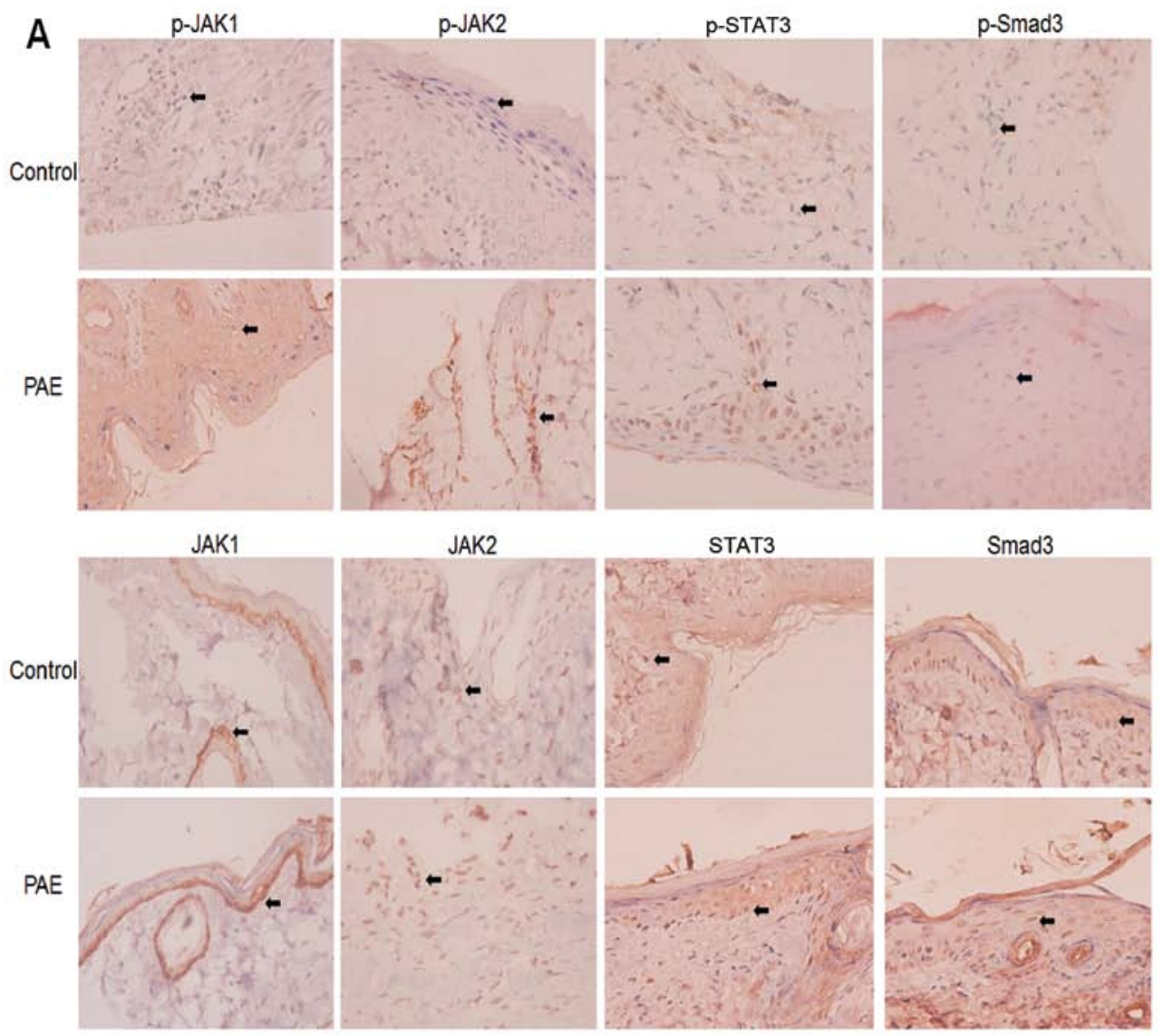

B
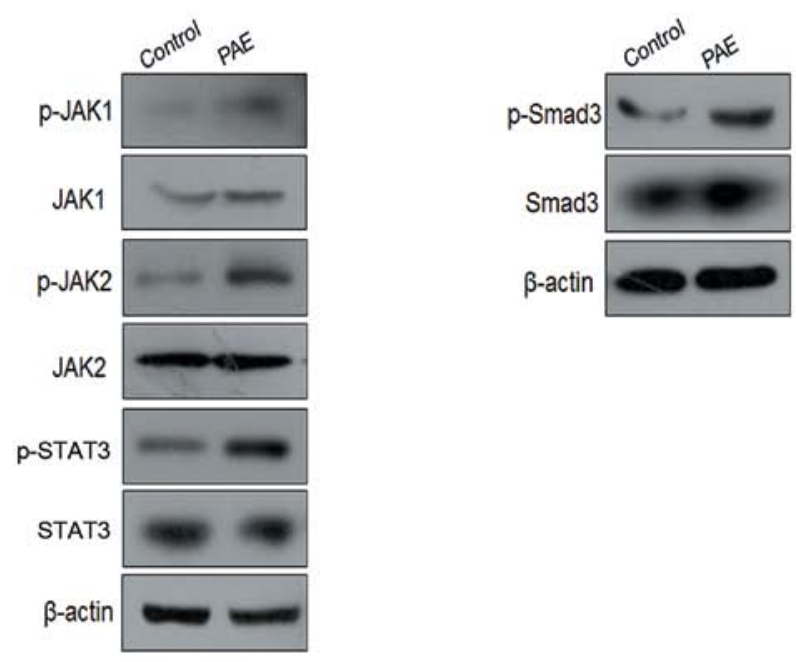

Figure 7. Effects of Periplaneta americana extract (PAE) on Janus-activated kinase/signal transducer and activator of transcription 3 (JAK/STAT3) signaling and Smad 3 activities in burn wound specimens in vivo. (A) Immunohistochemical (IHC) analysis of JAK/STAT3 signaling cascade, Smad3 and its phosphorylation in PAE-treated or control tissues. Representative images are shown (magnification, x400). (B) Western blotting of JAK/STAT3 signaling proteins, Smad 3 and its phosphorylation using $\beta$-actin as a loading control. Data are expressed as mean \pm SD. The arrows in (A) indicate p-JAK1/JAK1, p-JAK2/JAK2, p-STAT3/STAT3, p-Smad3/Smad3 expression in the nucleus and cytoplasm, respectively.

their dimerization and translocation into the nucleus whereas STATs act as transcription factors with pleiotropic downstream effects (34). The pivotal roles of JAK/STAT3 signaling and TGF- $\beta / \mathrm{Smad} 3$ in cutaneous wound healing are well known (15,35-37). The proliferation and differentiation of keratinocytes during wound healing are regulated by cytokines and chemokines, which are secreted by resident and inflammatory cells and activate the transcription factor
STAT3 $(38,39)$. Moreover, the specific ablation of STAT3 in the follicular and interfollicular keratinocytes resulted in impaired wound healing (24). Our results suggested that the enhanced phosphorylation and activation of JAK/STAT3 and Smad3 by the active ingredients in PAE accelerates wound healing efficiency. However, the composition of active ingredients and the downstream factors need to be defined to elucidate the mechanism of PAE. 
Consequently, PAE promoted wound healing in vitro and in vivo experimental models, which were strongly correlated with the activation of JAK/STAT3 and Smad3 signaling. Pretreatment with stattic inhibited $\mathrm{HaCaT}$ cell proliferation and migration induced by PAE. Furthermore, the enhanced activities of JAK/STAT3 and Smad3 pathways may represent major molecular targets of PAE, in accelerated wound healing.

\section{Acknowledgements}

This study was supported by the National Natural Science Foundation of China (no. 81403194).

\section{References}

1. Luo TS, Gao MT, Ma FF, Liu GM and Zhang CG: Research advances in pharmacological action and clinical application of Periplaneta americana. Agric Sci Technol 13: 888-892, 2012.

2. Chen XH, Ran XZ, Sun RS, Shi CM, Su Y, Guo CH and Cheng TM: Protective effect of an extract from Periplaneta americana on hematopoiesis in irradiated rats. Int $\mathbf{J}$ Radiat Biol 85: 607-613, 2009.

3. Yan MC, Yang H and Yang RM: Observation of the clinical effects of $\mathrm{W}_{11}-\mathrm{a}_{12}$. Yunnan Med 3: 138-140, 1987.

4. Pan XL: The observation and nurse care with Kangfuxin local medicine - change in treating diabetes acromelic gangrene. J Nurse Train 11: 26-27, 1996.

5. Wang HL and Liu J: Clinical observation of patients burned at III degree in small proportion of total surface area treated with Kangfuxin. J Binzhou Med Coll 22: 368, 1999.

6. Lin Q, Cao D, Yang YQ, et al: Study on action of Kangfuxin solution on experimental gastric ulcer. Chin Trad Pat Med 23: 122-124, 2001.

7. Du GM and Li WL: The clinical and laboratory studies about effects of Kangfuxin on superoxide dismutase and cell immune function of old persons. Yunnan J Chin Trad Med 10: 32-33, 1989.

8. Man HX, Huang L, Na KG, et al: Research progress of chemical component and biological activity in medicinal Periplaneta americana. Anti-infect Pharmacol 11: 403-407, 2014.

9. Wang ZY, Huang XH, Xie YK, Chen S and Wang S: Influence of Kangfuxin liquid on the wound healing of experimental animal burns and scalds. J Tradit Chin Med 52: 1316-1317, 2011.

10. Shu CX, Cheng TM and Yan GM: Effects of whole body irradiation on several components of skin wound extracellular matrix and the repair-promoting action of $\mathrm{W}_{11}-\mathrm{a}_{12}$. Chin $\mathrm{J}$ Traumatol 11: 604-607, 2001.

11. Shu CX, Ye BL, Cheng TM and Xiao JS: Effects of irradiation and $\mathrm{W}_{11}-\mathrm{a}_{12}$ on anion-selective channel of mouse peritoneal macrophage. Acta Acade Med Milita Tertiae 23: 290-292, 2001.

12. Chen XH, Cheng TM and Ai GP: Effects of systemic irradiation and $\mathrm{W}_{11}-\mathrm{a}_{12}$ on neutrophils in wounds. Acta Acade Med Milita Tertiae 23: 287-289, 2001.

13. Chen XH, Sun RS, Cheng TM, et al: Neutrophil apoptosis in wound of irradiated rats and effects of $\mathrm{W}_{11}-\mathrm{a}_{12}$ in accelerating wound healing. Chin J Clin Rehabil 9: 108-110, 2005.

14. Amadeu TP, Seabra AB, de Oliveira MG and MonteAlto-Costa A: Nitric oxide donor improves healing if applied on inflammatory and proliferative phase. J Surg Res 149: 84-93, 2008.

15. Li PN, Li H, Zhong LX, Sun Y, Yu LJ, Wu ML, Zhang LL, Kong QY, Wang SY and Lv DC: Molecular events underlying maggot extract promoted rat in vivo and human in vitro skin wound healing. Wound Repair Regen 23: 65-73, 2015.

16. Pakyari M, Farrokhi A, Maharlooei MK and Ghahary A: Critical role of transforming growth factor beta in different phases of wound healing. Adv Wound Care (New Rochelle) 2: 215-224, 2013.

17. Ren X, Ge M, Qin X, Xu P, Zhu P, Dang Y, Gu J and Ye X S100a8/NF- $\mathrm{kB}$ signal pathway is involved in the 800-nm diode laser-induced skin collagen remodeling. Lasers Med Sci 31: 673-678, 2016

18. Shi Y, Shu B, Yang R, Xu Y, Xing B, Liu J, Chen L, Qi S, Liu X, Wang $\mathrm{P}$, et al: Wnt and Notch signaling pathway involved in wound healing by targeting c-Myc and Hes1 separately. Stem Cell Res Ther 6: 120, 2015.
19. Tokumaru S, Sayama K, Yamasaki K, Shirakata Y, Hanakawa Y, Yahata Y, Dai X, Tohyama M, Yang L, Yoshimura A, et al: SOCS3/CIS3 negative regulation of STAT3 in HGF-induced keratinocyte migration. Biochem Biophys Res Commun 327: 100-105, 2005 .

20. Yasukawa H, Ohishi M, Mori H, Murakami M, Chinen T, Aki D, Hanada T, Takeda K, Akira S, Hoshijima M, et al: IL-6 induces an anti-inflammatory response in the absence of SOCS3 in macrophages. Nat Immunol 4: 551-556, 2003.

21. Lejeune D, Dumoutier L, Constantinescu S, Kruijer W, Schuringa JJ and Renauld JC: Interleukin-22 (IL-22) activates the JAK/STAT, ERK, JNK, and p38 MAP kinase pathways in a rat hepatoma cell line. Pathways that are shared with and distinct from IL-10. J Biol Chem 277: 33676-33682, 2002.

22. Wolk K, Witte E, Witte K, Warszawska K and Sabat R: Biology of interleukin-22. Semin Immunopathol 32: 17-31, 2010.

23. Yu R, Ding Y, Zhu L, Qu Y, Zhang C, Liu L and Chen L: IL-22 mediates the oral mucosal wound healing via STAT3 in keratinocytes. Arch Oral Biol 72: 14-20, 2016

24. Sano S, Itami S, Takeda K, Tarutani M, Yamaguchi Y, Miura H, Yoshikawa K, Akira S and Takeda J: Keratinocyte-specific ablation of Stat 3 exhibits impaired skin remodeling, but does not affect skin morphogenesis. EMBO J 18: 4657-4668, 1999.

25. Penn JW, Grobbelaar AO and Rolfe KJ: The role of the TGF- $\beta$ family in wound healing, burns and scarring: A review. Int J Burns Trauma 2: 18-28, 2012.

26. Hong HJ, Jin SE, Park JS, Ahn WS and Kim CK: Accelerated wound healing by smad 3 antisense oligonucleotides-impregnated chitosan/alginate polyelectrolyte complex. Biomaterials 29: 4831-4837, 2008.

27. Biernacka A, Dobaczewski $M$ and Frangogiannis NG: TGF- $\beta$ signaling in fibrosis. Growth Factors 29: 196-202, 2011.

28. Werner S, Krieg T and Smola H: Keratinocyte-fibroblast interactions in wound healing. J Invest Dermatol 127: 998-1008, 2007.

29. Tavares Pereira DS, Lima-Ribeiro MH, de Pontes-Filho NT, Carneiro-Leão AM and Correia MT: Development of animal model for studying deep second-degree thermal burns. J Biomed Biotechnol 2012: 460841, 2012.

30. Zhang J, La X, Fan L, Li P, Yu Y, Huang Y, Ding J and Xing Y: Immunosuppressive effects of mesenchymal stem cell transplantation in rat burn models. Int J Clin Exp Pathol 8: 5129-5136, 2015.

31. Diegelmann RF and Evans MC: Wound healing: An overview of acute, fibrotic and delayed healing. Front Biosci 9: 283-289, 2004.

32. Hata S, Okamura K, Hatta M, Ishikawa H and Yamazaki J: Proteolytic and non-proteolytic activation of keratinocytederived latent TGF- $\beta 1$ induces fibroblast differentiation in a wound-healing model using rat skin. J Pharmacol Sci 124: 230-243, 2014.

33. Yamaoka H, Sumiyoshi H, Higashi K, Nakao S, Minakawa K, Sumida K, Saito K, Ikoma N, Mabuchi T, Ozawa A, et al: A novel small compound accelerates dermal wound healing by modifying infiltration, proliferation and migration of distinct cellular components in mice. J Dermatol Sci 74: 204-213, 2014.

34. Levy DE and Darnell JE Jr: Stats: Transcriptional control and biological impact. Nat Rev Mol Cell Biol 3: 651-662, 2002.

35. Zhu BM, Ishida Y, Robinson GW, Pacher-Zavisin M, Yoshimura A, Murphy PM and Hennighausen L: SOCS3 negatively regulates the gp130-STAT3 pathway in mouse skin wound healing. J Invest Dermatol 128: 1821-1829, 2008.

36. Murray PJ: The JAK-STAT signaling pathway: Input and output integration. J Immunol 178: 2623-2629, 2007.

37. Tokumaru S, Sayama K, Shirakata Y, Komatsuzawa H, Ouhara K, Hanakawa Y, Yahata Y, Dai X, Tohyama M, Nagai H, et al: Induction of keratinocyte migration via transactivation of the epidermal growth factor receptor by the antimicrobial peptide LL-37. J Immunol 175: 4662-4668, 2005.

38. Avitabile S, Odorisio T, Madonna S, Eyerich S, Guerra L, Eyerich K, Zambruno G, Cavani A and Cianfarani F: Interleukin-22 promotes wound repair in diabetes by improving keratinocyte pro-healing functions. J Invest Dermatol 135: 28622870,2015

39. Nelson AM, Katseff AS, Ratliff TS and Garza LA: Interleukin 6 and STAT3 regulate p63 isoform expression in keratinocytes during regeneration. Exp Dermatol 25: 155-157, 2016. 